\title{
An Extension of the Chebyshev Polynomials
}

\author{
Anna Tatarczak ${ }^{1}$
}

Received: 22 February 2015 / Accepted: 4 October 2015 / Published online: 13 October 2015

(C) The Author(s) 2015. This article is published with open access at Springerlink.com

\begin{abstract}
Our consideration is focused on determining properties of generalized Chebyshev polynomials of the first and second kind, sparking interest in constructing a theory similar to the classical one. This studies highlight some important results and connections between this two types. The paper is also concerned with the connection between orthogonal polynomials and typically real function, both strictly related to the Koebe function.
\end{abstract}

Keywords Orthogonal polynomials - Chebyshev polynomials · Bernstein type inequalities · Holomorphic functions · Typically real functions

\section{Introduction}

The classical Chebyshev polynomials of the first and second kind have been known since the late eighteenth century, when was defined using de Moivre's formula by Chebyshev [5]. In the study of differential equations they arise as the solution to the Chebyshev differential equations

$$
\left(1-x^{2}\right) y^{\prime \prime}-x y^{\prime}+n^{2} y=0,
$$

Communicated by Marek Bozejko.

$凶$ Anna Tatarczak

antatarczak@gmail.com; anna.tatarczak@ poczta.umcs.lublin.pl

1 Department of Mathematics, Maria Curie-Skłodowska University in Lublin, Lublin, Poland 
and

$$
\left(1-x^{2}\right) y^{\prime \prime}-3 x y^{\prime}+n(n+2) y=0,
$$

for the polynomials of the first and second kind, respectively. They are the special cases of the Sturm Liouville differential equation. Two properties of Chebyshev polynomials make them exceptionally suitable for approximations: monic Chebyshev polynomials minimize all norms among monic polynomials of a given degre, and satisfy discrete orthogonality relation. Based on these properties they are widely used in many areas of numerical analysis; uniform approximation, least-squares approximation, numerical solution of ordinary and partial differential equations, and so on. Therefore several its extensions occur, see Akhiezer [1,2], and Akhiezer and Tomčuk [3], Tomčuk [32], Ismail [14], Peherstorfer [24] and many others. The Chebyshev polynomials are orthogonal not only as polynomials in real variable but also as polynomials in a complex variable $z$ on elliptical contours and domains of the complex plane (the foci of the ellipses being \pm 1 ). This property is exploited in fields that rely on complex variable techniques. Later on polynomials which are not fully in agreement with orthogonal polynomials, hence called the (generalized) Chebyshev type polynomials appeared, see for example Peherstorfer [24]. The Chebyshev type polynomials satisfy similar extremal properties to the classical Chebyshev polynomials on $[-1,1]$. The extremal polynomials also have the property that they are orthogonal with respect to some weight function. For other generalization the reader is referred to [6]. Some of generalized Chebyshev type polynomials are associated with generalized Koebe function, as was observed in [17].

In this paper, we will explore the sequences of polynomials of the generalized Chebyshev polynomial of the second kind $U_{n}\left(p, q ; e^{i \theta}\right)$ and of the first kind $T_{n}\left(p, q ; e^{i \theta}\right)$. Each of these sequences is useful in applications for a particular reason. The Chebyshev polynomials of second kind are defined by the fact its connections with the generalized typically real functions; similarly as was in the classical case. The coefficient problem for generalized typically real functions provides one motivation to study properties of Chebyshev polynomials. This work highlights the connections between this two types of orthogonal polynomials.

\section{Basic Definition}

The impact of the work Russian mathematician P. L. Chebyshev (1821-1894) and his studen Markov has already been describe by Krein [20]. A few particular orthogonal polynomials were known before Chebyschev. It was Chebyshev who saw the possiblity of a general theory and its applications. His work arose out of the theory of least squares approximation and probability. He discovered the discrete analogue of the Jacobi polynomials but their importance was not recognized until twentieth century. They were rediscovered by Hahn and named after him upon their rediscovered. Nowadays the notion of Chebyshev polynomials is well known [7,31]. The sequence of polynomials $\left\{T_{n}(x)\right\}_{n=0}^{\infty}$ appearing in approximation theory [25], geometry [11], combinatorics [29], number theory [8], statistics, numerical integration [9,22], and 
differential equations (Rivlin [26] gives numerous examples). Several generalizations have been found and investigated, see e.g. [30,33].

For easy reference, let us first state the definitions and basic properties of Chebyshev polynomials.

Definition 1 The fundamental Chebyshev polynomials of the first kind are defined by the following recurrence relation

$$
\begin{aligned}
& T_{0}(x)=1 \\
& T_{1}(x)=x \\
& T_{n}(x)=2 x T_{n-1}(x)-T_{n-2}(x), \quad-1 \leq x \leq 1, \quad n \geq 2 .
\end{aligned}
$$

Definition 2 The Chebyshev polynomials of the second kind are defined by the recurrence relation

$$
\begin{aligned}
& U_{0}(x)=1, \\
& U_{1}(x)=2 x \\
& U_{n}(x)=2 x U_{n-1}(x)-U_{n-2}(x), \quad-1 \leq x \leq 1, \quad n \geq 2 .
\end{aligned}
$$

First generalization of Chebyshev polynomials of first kind we met in [17], where it was proposed to study the polynomials with one parameter, namely

$$
T_{n}\left(q ; e^{i \theta}\right)=\frac{1}{2}\left(e^{i n \theta}+q^{n} e^{-i n \theta}\right), \quad q \in[-1,1]
$$

Next in [23] some properties of the generalized Chebyshev polynomials $U_{n}\left(p, q ; e^{i \theta}\right)$ of the second kind was studied. These polynomials were defined by the generating function.

Definition 3 Let $\theta \in[0,2 \pi],-1 \leq p, q \leq 1$. The generalized Chebyshev polynomials of the second kind $U_{n}\left(p, q ; e^{i \theta}\right)$ are defined by

$$
\begin{aligned}
\Psi^{(p, q)}\left(e^{i \theta} ; z\right) & =\frac{1}{\left(1-p z e^{i \theta}\right)\left(1-q z e^{-i \theta}\right)} \\
& =\sum_{n=0}^{\infty} U_{n}\left(p, q ; e^{i \theta}\right) z^{n} \quad(z \in \mathbb{D}),
\end{aligned}
$$

where $\theta \in[0,2 \pi],-1 \leq p, q \leq 1$, or by an explicit formulas

$$
\begin{aligned}
U_{0}\left(p, q ; e^{i \theta}\right) & =1, \\
U_{1}\left(p, q ; e^{i \theta}\right) & =p e^{i \theta}+q e^{-i \theta}, \\
U_{n}\left(p, q ; e^{i \theta}\right) & =\frac{p^{n+1} e^{i(n+1) \theta}-q^{n+1} e^{-i(n+1) \theta}}{p e^{i \theta}-q e^{-i \theta}} \quad(n \geq 2) .
\end{aligned}
$$


In [23] it was also proposed to study some generalization of the Chebyshev polynomials of the first kind, namely.

Definition 4 Let $\theta \in[0,2 \pi],-1 \leq p, q \leq 1$. The generalized Chebyshev polynomials of the first kind are defined by

$$
T_{n}\left(p, q ; e^{i \theta}\right)=\frac{1}{2}\left(p^{n} e^{i n \theta}+q^{n} e^{-i n \theta}\right) \quad(n=0,1, \ldots)
$$

where $\theta \in[0,2 \pi],-1 \leq p, q \leq 1$.

\section{The Main Properties of Polynomials $T_{n}\left(p, q ; e^{i \theta}\right)$ and $U_{n}\left(p, q ; e^{i \theta}\right)$}

In this section we present the main properties of the introduced polynomials (2.5).

Theorem 1 Let $\theta \in[-\pi, \pi],(p, q) \in \Delta=\{(p, q):-1 \leq q \leq p \leq 1\}$. Then the following relations hold.

(a) The trigonometric polynomials $T_{n}\left(p, q ; e^{i \theta}\right)$ satisfy the three-term recurrence relation

$$
\begin{aligned}
T_{0}\left(p, q ; e^{i \theta}\right) & =1 \\
T_{1}\left(p, q ; e^{i \theta}\right) & =\frac{1}{2}\left(p e^{i \theta}+q e^{-i \theta}\right) \\
T_{n+2}\left(p, q ; e^{i \theta}\right)= & \left(p e^{i \theta}+q e^{-i \theta}\right) T_{n+1}\left(p, q ; e^{i \theta}\right)-p q T_{n}\left(p, q ; e^{i \theta}\right) \\
& n=0,1, \ldots
\end{aligned}
$$

(b) The function $y(\theta)=T_{n}\left(p, q ; e^{i \theta}\right)$, satisfies the following differential equation of the second order

$$
y^{\prime \prime}(\theta)+n^{2} y(\theta)=0 .
$$

(c) The trigonometric polynomials $T_{n}\left(p, q ; e^{i \theta}\right)$ satisfy the following orthogonality relation

$$
\int_{-\pi}^{\pi} T_{n}\left(p, q ; e^{i \theta}\right) \overline{T_{m}}\left(p, q ; e^{i \theta}\right) d \theta= \begin{cases}0 & \text { if } m \neq n \\ \frac{\pi}{2}\left(p^{2 n}+q^{2 n}\right) & \text { if } m=n \neq 0 \\ 2 \pi & \text { if } m=n=0\end{cases}
$$

(d) The generating function of $\left\{T_{n}\left(p, q ; e^{i \theta}\right)\right\}_{n \geq 0}$ has the form

$$
\sum_{n=0}^{\infty} T_{n}\left(p, q ; e^{i \theta}\right) z^{n}=\frac{1-\frac{\left(p e^{i \theta}+q e^{-i \theta}\right)}{2} z}{\left(1-p e^{i \theta} z\right)\left(1-q e^{-i \theta} z\right)}
$$

where $z \in \mathbb{D}$. 
(e)

$$
\max _{-\pi \leq \theta \leq \pi}\left|T_{n}\left(p, q ; e^{i \theta}\right)\right|=\frac{1}{2} \sqrt{p^{2 n}+q^{2 n}+2(p q)^{n}} .
$$

Proof (a) Setting $n=0$ and $n=1$ in (2.5) we get the first two equalities of (a).

Now we put

$$
2 T_{n}\left(p, q ; e^{i \theta}\right)=p^{n} e^{i n \theta}+q^{n} e^{-i n \theta}
$$

Therefore

$$
2 T_{n+1}\left(p, q ; e^{i \theta}\right)=p^{n+1} e^{i(n+1) \theta}+q^{n+1} e^{-i(n+1) \theta},
$$

and

$$
2 T_{n+2}\left(p, q ; e^{i \theta}\right)=p^{n+2} e^{i(n+2) \theta}+q^{n+2} e^{-i(n+2) \theta} .
$$

First we multiplying (3.6) by $p q$ and (3.7) by $p e^{i \theta}+q e^{-i \theta}$. Afterwards we get the desired formula by substracting $p q T_{n}\left(p, q ; e^{i \theta}\right)$ from $\left(p e^{i \theta}+\right.$ $\left.q e^{-i \theta}\right) T_{n+1}\left(p, q ; e^{i \theta}\right)$.

(b) Using the explicit formula (2.5), after double differentiation we obtain the desired result.

(c) The scalar product of $T_{n}\left(p, q ; e^{i \theta}\right)$ and $T_{m}\left(p, q ; e^{i \theta}\right)$ equals

$$
\begin{aligned}
\left\langle T_{n}, T_{m}\right\rangle= & \int_{-\pi}^{\pi} T_{n}\left(p, q ; e^{i \theta}\right) \overline{T_{m}}\left(p, q ; e^{i \theta}\right) d \theta \\
= & \frac{1}{4} \int_{-\pi}^{\pi}\left(p^{n+m} e^{i(n-m) \theta}+p^{n} q^{m} e^{i(n+m) \theta}+q^{n} p^{m} e^{-i(n+m) \theta}\right. \\
& \left.+q^{n+m} e^{-i(n-m) \theta}\right) d \theta .
\end{aligned}
$$

Since

$$
\int_{-\pi}^{\pi} e^{i m \theta}= \begin{cases}0 & \text { if } m \neq 0 \\ 2 \pi & \text { if } m=0\end{cases}
$$

then we easily see that $\left\langle T_{m}, T_{n}\right\rangle$ is expressed by (3.3).

(d) By (2.5) we have

$$
\begin{aligned}
\sum_{n=0}^{\infty} T_{n}\left(p, q ; e^{i \theta}\right) z^{n} & =\frac{1}{2} \sum_{n=0}^{\infty}\left(p^{n} e^{i n \theta}+q^{n} e^{-i n \theta}\right) z^{n} \\
& =\frac{1}{2} \sum_{n=0}^{\infty}\left(p e^{i \theta} z\right)^{n}+\frac{1}{2} \sum_{n=0}^{\infty}\left(q e^{-i \theta} z\right)^{n} \\
& =\frac{1}{2} \frac{1}{1-p e^{i \theta} z}+\frac{1}{2} \frac{1}{1-q e^{-i \theta} z} \\
& =\frac{1-\frac{\left(p e^{i \theta}+q e^{-i \theta}\right)}{2} z}{\left(1-p e^{i \theta} z\right)\left(1-q e^{-i \theta} z\right)} .
\end{aligned}
$$


(e) Straightford calculation of (2.5) gives

$$
\begin{aligned}
\left|T_{n}\left(p, q ; e^{i \theta}\right)\right| & =\sqrt{\frac{1}{4}\left(p^{n}+q^{n}\right)^{2} \cos ^{2} n \theta+\frac{1}{4}\left(p^{n}-q^{n}\right)^{2} \sin ^{2} n \theta} \\
& =\frac{1}{2} \sqrt{p^{2 n}+q^{2 n}+2 p^{n} q^{n} \cos 2 n \theta}
\end{aligned}
$$

From which we get the desired result.

Note that the norm of $\left\{T_{n}\left(p, q ; e^{i \theta}\right\}\right.$ is given by

$$
\left\|T_{n}\left(p, q ; e^{i \theta}\right)\right\|^{2}=\int_{-\pi}^{\pi} T_{n}\left(p, q ; e^{i \theta}\right) \overline{T_{n}}\left(p, q ; e^{i \theta}\right) d \theta=\frac{\pi\left(p^{2 n}+q^{2 n}\right)}{2},
$$

and

$$
\left\|T_{0}\left(p, q ; e^{i \theta}\right)\right\|^{2}=2 \pi
$$

The system $\left\{T_{n}\left(p, q ; e^{i \theta}\right)\right\}$ is therefore not ortonormal. By introducing the respective weight we find the ortonormal system:

$$
\left\{\frac{T_{0}\left(p, q ; e^{i \theta}\right)}{\sqrt{2 \pi}},\left(\sqrt{\frac{2}{\pi\left(p^{2 n}+q^{2 n}\right)}} T_{n}\left(p, q ; e^{i \theta}\right), \quad n=1,2, \ldots\right)\right\} .
$$

It is well known that the classical Chebyshev polynomials $U_{n}(x)$ and $T_{n}(x)$ are connected by several relations (see [4]). We observe that similar relations between $U_{n}\left(p, q ; e^{i \theta}\right)$ and $T_{n}\left(p, q ; e^{i \theta}\right)$ also holds.

Proposition 1 For polynomials $U_{n}\left(p, q ; e^{i \theta}\right)$ and $T_{n}\left(p, q ; e^{i \theta}\right)$, where $\theta \in[-\pi, \pi]$, $(p, q) \in \Delta$, we have

$$
\begin{aligned}
T_{n}\left(p, q ; e^{i \theta}\right) T_{m}\left(p, q ; e^{i \theta}\right) & =\frac{1}{2} T_{n+m}\left(p, q ; e^{i \theta}\right)+\frac{1}{2}(p q)^{m} T_{n-m}\left(p, q ; e^{i \theta}\right) \\
& =\frac{1}{2} T_{n+m}\left(p, q ; e^{i \theta}\right)+\frac{1}{2}(p q)^{n} T_{m-n}\left(p, q ; e^{i \theta}\right) . \\
U_{m-1}\left(T_{n}\right) & =\frac{2 p^{m} T_{2 n}\left(p, q ; e^{i \theta}\right)+2 p^{n+m} q^{n}-q^{m}}{2 T_{n}\left(p, q ; e^{i \theta}\right)\left(p e^{i \theta}+q e^{-i \theta}\right)} .
\end{aligned}
$$

Remark 1 The trigonometric polynomials $U_{n}\left(p, q ; e^{i \theta}\right)$ and $T_{n}\left(p, q ; e^{i \theta}\right)$, where $\theta \in$ $[-\pi, \pi],(p, q) \in \Delta$, have the following representation:

$$
\begin{aligned}
U_{n}\left(p, q ; e^{i \theta}\right) & =\sum_{k=0}^{[n / 2]}(-p q)^{k} \frac{(n-k) !}{(n-2 k) ! k !}\left(p e^{i \theta}+q e^{-i \theta}\right)^{n-2 k}, \\
T_{n}\left(p, q ; e^{i \theta}\right) & =\frac{n}{2} \sum_{k=0}^{[n / 2]}(-p q)^{k} \frac{(n-k-1) !}{(n-2 k) ! k !}\left(p e^{i \theta}+q e^{-i \theta}\right)^{n-2 k} .
\end{aligned}
$$


Remark 2 One can show that

$$
T_{n}\left(p, q ;-e^{i \theta}\right)=(-1)^{n} T_{n}\left(p, q ; e^{i \theta}\right)
$$

or in other words, the even degree Chebyshev polynomials are even functions and the odd Chebyshev polynomials are odd functions.

Remark 3 Putting the value $\frac{z}{\sqrt{p q}}$, with $p q \neq 0$ instead of $z$ in the generating function (3.4) and comparing the result with the generating function for $T_{n}(x)$ we conclude that

$$
T_{n}\left(p, q ; e^{i \theta}\right)=(\sqrt{p q})^{n} T_{n}\left(\frac{p e^{i \theta}+q e^{-i \theta}}{2 \sqrt{p q}}\right), \quad p q \neq 0 .
$$

Observe that, if $\theta \in[-\pi, \pi]$, then $\omega=\omega(\theta)$ given by

$$
\omega(\theta)=\frac{p e^{i \theta}+q e^{-i \theta}}{2 \sqrt{p q}}
$$

describes the ellipse $E$ with semi-axes: $a=\left|\frac{(p+q)}{2 \sqrt{p q}}\right|$ and $b=\left|\frac{(p-q)}{2 \sqrt{p q}}\right|$.

Remark 4 Note that for $\theta=0$ and $(p, q) \in \Delta, p \neq q, n \geq 1$ in (2.4) and (2.5) we have

$$
\begin{aligned}
U_{n-1}(p, q ; 1) & =\frac{p^{n}-q^{n}}{p-q}, \\
T_{n}(p, q ; 1) & =\frac{p^{n}+q^{n}}{2} .
\end{aligned}
$$

From (3.9) we immediately have relation between $T_{n}(p, q ; 1)$ and $U_{n}(p, q ; 1)$.

$$
\begin{aligned}
2 T_{n}(p, q ; 1) & =p^{n}+q^{n}=\frac{p^{n-1}-q^{n-1}}{p-q}+\frac{p^{n+1}-q^{n+1}}{p-q} \\
& =U_{n-2}(p, q ; 1)+U_{n}(p, q ; 1) \\
2 T_{n}(p, q ; 1) & =p^{n}+q^{n}=\frac{\frac{p^{2 n}-q^{2 n}}{p-q}}{\frac{p^{n}-q^{n}}{p-q}} \\
& =\frac{U_{2 n-1}(p, q ; 1)}{U_{n-1}(p, q ; 1)}
\end{aligned}
$$

\section{The Products, Integrals and Derivatives of Generalized Chebyshev Polynomials}

It is well known that the classical Chebyshev polynomials satisfy several equalities, that involve factor $x$ or $1-x^{2}$ (where $x=\cos \theta$ see, for instance [21]). Also the 
product of both polynomials $T_{n}(x)$ and $U_{n}(x)$ where considered [21]. Let us remain the well know relation for $T_{n}(x), U_{n}(x)$ below.

$$
\begin{aligned}
& x T_{n}(x)=\frac{1}{2}\left(T_{n+1}(x)+T_{|n-1|}(x)\right), \\
& x U_{n}(x)=\frac{1}{2}\left(U_{n+1}(x)+U_{|n-1|}(x)\right), \\
& \left(1-x^{2}\right) T_{n}(x)=-\frac{1}{4} T_{n+2}(x)+\frac{1}{2} T_{n}(x)-\frac{1}{4} T_{|n-2|}(x), \\
& \left(1-x^{2}\right) U_{n}(x)=-\frac{1}{4} U_{n+2}(x)+\frac{1}{2} U_{n}(x)-\frac{1}{4} U_{|n-2|}(x), \\
& \int T_{n}(x) d x= \begin{cases}\frac{1}{2}\left(\frac{T_{n+1}(x)}{n+1}-\frac{T_{|n-1|}}{n-1}\right), & n \neq 1, \\
\frac{1}{4} T_{2}(x), & n=1,\end{cases} \\
& \int U_{n}(x) d x=\frac{1}{n+1} T_{n+1}(x)+\text { Const }, \\
& \frac{d}{d x} T_{n}(x)=\frac{n}{2} \frac{T_{n-1}(x)-T_{n+1}(x)}{1-x^{2}}, \\
& \frac{d}{d x} U_{n}(x)=\frac{(n+2) U_{n-1}(x)-n U_{n+1}(x)}{2\left(1-x^{2}\right)} .
\end{aligned}
$$

In this section we formulate similar properties for generalized Chebyshev polynomials $T_{n}\left(p, q ; e^{i \theta}\right)$ and $U_{n}\left(p, q ; e^{i \theta}\right)$, below.

Proposition 2 Let $T_{n}\left(p, q ; e^{i \theta}\right)$ and $U_{n}\left(p, q ; e^{i \theta}\right)$ be defined by (2.4) and (3.4). Then

$$
\begin{aligned}
& \cos \theta T_{n}\left(p, q ; e^{i \theta}\right) \\
&= T_{n}(p, q ; 1)\left(\frac{\operatorname{Re} T_{n+1}\left(p, q ; e^{i \theta}\right)}{T_{n+1}(p, q ; 1)}+\frac{\operatorname{Re} T_{|n-1|}\left(p, q ; e^{i \theta}\right)}{T_{|n-1|}(p, q ; 1)}\right) \\
&+U_{|n-1|}(p, q ; 1)\left(\frac{i \operatorname{Im} T_{n+1}\left(p, q ; e^{i \theta}\right)}{U_{n}(p, q ; 1)}+\frac{i \operatorname{Im} T_{|n-1|}\left(p, q ; e^{i \theta}\right)}{U_{|n-2|}(p, q ; 1)}\right) . \\
&\left(1-\cos ^{2} \theta\right) T_{n}\left(p, q ; e^{i \theta}\right)=\frac{1}{2} T_{n}\left(p, q ; e^{i \theta}\right)-\frac{1}{4} T_{n}(p, q ; 1) \\
& \quad \times\left(\frac{\operatorname{Re} T_{n+2}\left(p, q ; e^{i \theta}\right)}{T_{n+2}(p, q ; 1)}+\frac{\operatorname{Re} T_{|n-2|}\left(p, q ; e^{i \theta}\right)}{T_{|n-2|}(p, q ; 1)}\right) \\
& \quad-\frac{1}{4} U_{|n-1|}(p, q ; 1)\left(\frac{i \operatorname{Im} T_{n+2}\left(p, q ; e^{i \theta}\right)}{U_{n+1}(p, q ; 1)}+\frac{i \operatorname{Im} T_{|n-2|}\left(p, q ; e^{i \theta}\right)}{U_{|n-3|}(p, q ; 1)}\right) .
\end{aligned}
$$


Proof In order to proof (4.1) consider its left hand side and apply (2.5). Then we have

$$
\begin{aligned}
\cos & \theta T_{n}\left(p, q ; e^{i \theta}\right) \\
= & \cos \theta\left(\frac{1}{2}\left(p^{n}+q^{n}\right) \cos n \theta+\frac{1}{2} i\left(p^{n}-q^{n}\right) \sin n \theta\right) \\
= & \frac{1}{2}\left(p^{n}+q^{n}\right) \cos (n+1) \theta+\frac{1}{2}\left(p^{n}+q^{n}\right) \cos (n-1) \theta \\
& +\frac{1}{2} i\left(p^{n}-q^{n}\right) \sin (n+1) \theta+\frac{1}{2} i\left(p^{n}-q^{n}\right) \sin (n-1) \theta \\
= & \left(p^{n}+q^{n}\right)\left(\frac{\operatorname{Re} T_{n+1}\left(p, q ; e^{i \theta}\right)}{p^{n+1}+q^{n+1}}+\frac{\operatorname{Re} T_{|n-1|}\left(p, q ; e^{i \theta}\right)}{p^{n-1}+q^{n-1}}\right) \\
& +\left(p^{n}-q^{n}\right)\left(\frac{i \operatorname{Im} T_{n+1}\left(p, q ; e^{i \theta}\right)}{p^{n+1}-q^{n+1}}+\frac{i \operatorname{Im} T_{|n-1|}\left(p, q ; e^{i \theta}\right)}{p^{n-1}-q^{n-1}}\right) \\
= & T_{n}(p, q ; 1)\left(\frac{\operatorname{Re} T_{n+1}\left(p, q ; e^{i \theta}\right)}{T_{n+1}(p, q ; 1)}+\frac{\operatorname{Re} T_{|n-1|}\left(p, q ; e^{i \theta}\right)}{T_{|n-1|}(p, q ; 1)}\right) \\
& +U_{|n-1|}(p, q ; 1)\left(\frac{i \operatorname{Im} T_{n+1}\left(p, q ; e^{i \theta}\right)}{U_{n}(p, q ; 1)}+\frac{i \operatorname{Im} T_{|n-1|}\left(p, q ; e^{i \theta}\right)}{U_{|n-2|}(p, q ; 1)}\right) .
\end{aligned}
$$

Similarly we proof the equality (4.2):

$$
\begin{aligned}
(1 & \left.-\cos ^{2} \theta\right) T_{n}\left(p, q ; e^{i \theta}\right)=\sin ^{2} \theta\left(\frac{1}{2}\left(p^{n}+q^{n}\right) \cos n \theta+\frac{1}{2} i\left(p^{n}-q^{n}\right) \sin n \theta\right) \\
= & \frac{1}{2}(1-\cos 2 \theta) T_{n}\left(p, q ; e^{i \theta}\right) \\
= & \frac{1}{2} T_{n}\left(p, q ; e^{i \theta}\right)-\frac{1}{4}\left(p^{n}+q^{n}\right)\left(\frac{\operatorname{Re} T_{n+2}\left(p, q ; e^{i \theta}\right)}{p^{n+2}+q^{n+2}}+\frac{\operatorname{Re} T_{|n-2|}\left(p, q ; e^{i \theta}\right)}{p^{n-2}+q^{n-2}}\right) \\
& -\frac{1}{4}\left(p^{n}-q^{n}\right)\left(\frac{i \operatorname{Im} T_{n+2}\left(p, q ; e^{i \theta}\right)}{p^{n+2}-q^{n+2}}+\frac{i \operatorname{Im} T_{|n-2|}\left(p, q ; e^{i \theta}\right)}{p^{n-2}-q^{n-2}}\right) \\
= & \frac{1}{2} T_{n}\left(p, q ; e^{i \theta}\right)-\frac{1}{4} T_{n}(p, q ; 1)\left(\frac{\operatorname{Re} T_{n+2}\left(p, q ; e^{i \theta}\right)}{T_{n+2}(p, q ; 1)}+\frac{\operatorname{Re} T_{|n-2|}\left(p, q ; e^{i \theta}\right)}{T_{|n-2|}(p, q ; 1)}\right) \\
& -\frac{1}{4} U_{|n-1|}(p, q ; 1)\left(\frac{i \operatorname{Im} T_{n+2}\left(p, q ; e^{i \theta}\right)}{U_{n+1}(p, q ; 1)}+\frac{i \operatorname{Im} T_{|n-2|}\left(p, q ; e^{i \theta}\right)}{U_{|n-3|}(p, q ; 1)}\right) .
\end{aligned}
$$

Proposition 3 Let $T_{n}\left(p, q ; e^{i \theta}\right)$ be defined by (2.5). Then the indefinite integral of $T_{n}(x)$ can be expressed in terms of Chebyshev polynomials as follows.

$$
\begin{aligned}
& \int T_{n}\left(p, q ; e^{i \theta}\right) d x \\
& =T_{n}(p, q ; 1)\left(\frac{\operatorname{Re} T_{n+1}\left(p, q ; e^{i \theta}\right)}{T_{n+1}(p, q ; 1)(n+1)}-\frac{\operatorname{Re} T_{|n-1|}\left(p, q ; e^{i \theta}\right)}{T_{|n-1|}(p, q ; 1)|n-1|}\right)
\end{aligned}
$$




$$
\begin{aligned}
& -U_{n-1}(p, q ; 1) i\left(\frac{\operatorname{Im} T_{n+1}\left(p, q ; e^{i \theta}\right)}{U_{n}(p, q ; 1)(n+1)}-\frac{\operatorname{Im} T_{|n-1|}\left(p, q ; e^{i \theta}\right)}{U_{|n-2|}(p, q ; 1)|n-1|}\right), \quad n \neq 1 \\
& \int T_{1}\left(p, q ; e^{i \theta}\right) d x=-i T_{1}\left(p, q ; e^{i \theta}\right) .
\end{aligned}
$$

Proof Applying the formula (2.5) by integration we obtain:

$$
\begin{aligned}
\int & T_{n}\left(p, q ; e^{i \theta}\right) d x \\
= & -\int \frac{1}{2}\left(p^{n}+q^{n}\right) \cos n \theta \sin \theta d \theta-i \int \frac{1}{2}\left(p^{n}-q^{n}\right) \sin n \theta \sin \theta d \theta \\
= & \frac{1}{2}\left(p^{n}+q^{n}\right) \frac{\cos (n+1) \theta}{n+1}-\frac{1}{2}\left(p^{n}+q^{n}\right) \frac{\cos |n-1| \theta}{|n-1|} \\
& -i \frac{1}{2}\left(p^{n}-q^{n}\right) \frac{\sin (n+1) \theta}{n+1}+\frac{1}{2} i\left(p^{n}-q^{n}\right) \frac{\sin |n-1| \theta}{|n-1|} \\
= & \left(p^{n}+q^{n}\right)\left(\frac{\operatorname{Re} T_{n+1}\left(p, q ; e^{i \theta}\right)}{\left(p^{n+1}+q^{n+1}\right)(n+1)}-\frac{\operatorname{Re} T_{|n-1|}\left(p, q ; e^{i \theta}\right)}{\left(p^{n-1}+q^{n-1}\right)|n-1|}\right) \\
& -\left(p^{n}-q^{n}\right) i\left(\frac{\operatorname{Im} T_{n+1}\left(p, q ; e^{i \theta}\right)}{\left(p^{n+1}-q^{n+1}\right)(n+1)}-\frac{\operatorname{Im} T_{|n-1|}\left(p, q ; e^{i \theta}\right)}{\left(p^{n-1}-q^{n-1}\right)|n-1|}\right) \\
= & T_{n}(p, q ; 1)\left(\frac{\operatorname{Re} T_{n+1}\left(p, q ; e^{i \theta}\right)}{T_{n+1}(p, q ; 1)(n+1)}-\frac{\operatorname{Re} T_{|n-1|}\left(p, q ; e^{i \theta}\right)}{T_{|n-1|}(p, q ; 1)|n-1|}\right) \\
& -U_{n-1}(p, q ; 1) i\left(\frac{\operatorname{Im} T_{n+1}\left(p, q ; e^{i \theta}\right)}{U_{n}(p, q ; 1)(n+1)}-\frac{\operatorname{Im} T_{|n-1|}\left(p, q ; e^{i \theta}\right)}{U_{|n-2|}(p, q ; 1)|n-1|}\right)
\end{aligned}
$$

(where the first term in the bracket is to be omitted in the case $n=1$ ).

Proposition 4 Let $T_{n}\left(p, q ; e^{i \theta}\right)$ be defined by (2.5), then we have

$$
\begin{aligned}
\frac{d}{d \theta} T_{n}\left(p, q ; e^{i \theta}\right)= & \frac{T_{n}(p, q ; 1)}{\sin ^{2} \theta}\left(\frac{n \operatorname{Re} T_{|n-1|}\left(p, q ; e^{i \theta}\right)}{2 T_{|n-1|}(p, q ; 1)}-\frac{\operatorname{Re} T_{n+1}\left(p, q ; e^{i \theta}\right)}{T_{n+1}(p, q ; 1)}\right) \\
& -\frac{U_{|n-1|}(p, q ; 1)}{\sin ^{2} \theta} i\left(\frac{n \operatorname{Im} T_{|n-1|}\left(p, q ; e^{i \theta}\right)}{2 U_{|n-2|}(p, q ; 1)}-\frac{\operatorname{Im} T_{n+1}\left(p, q ; e^{i \theta}\right)}{U_{n}(p, q ; 1)}\right) .
\end{aligned}
$$

Proof Differentiation of (2.5) we have:

$$
\begin{aligned}
\frac{d}{d \theta} T_{n}\left(p, q ; e^{i \theta}\right)= & \frac{d}{d \theta} \frac{1}{2}\left(p^{n}+q^{n}\right) \cos n \theta / \frac{d}{d \theta} \cos \theta \\
& +\frac{d}{d \theta} i \frac{1}{2}\left(p^{n}-q^{n}\right) \sin n \theta / \frac{d}{d \theta} \cos \theta \\
= & \frac{1}{2}\left(p^{n}+q^{n}\right) \frac{n \sin n \theta}{\sin \theta}-\frac{1}{2} i\left(p^{n}-q^{n}\right) \frac{n \cos n \theta}{\sin \theta}
\end{aligned}
$$




$$
\begin{aligned}
= & \frac{1}{2}\left(p^{n}+q^{n}\right) \frac{\frac{1}{2} n(\cos (n-1) \theta-\cos (n+1) \theta)}{\sin ^{2} \theta} \\
& -\frac{1}{2} i\left(p^{n}-q^{n}\right) \frac{\frac{1}{2} n(\sin (n-1) \theta-\sin (n+1) \theta)}{\sin ^{2} \theta} \\
= & \frac{p^{n}+q^{n}}{\sin ^{2} \theta}\left(\frac{n \operatorname{Re} T_{|n-1|}\left(p, q ; e^{i \theta}\right)}{2\left(p^{n-1}+q^{n-1}\right)}-\frac{\operatorname{Re}_{n+1}\left(p, q ; e^{i \theta}\right)}{p^{n+1}+q^{n+1}}\right) \\
& -\frac{p^{n}-q^{n}}{\sin ^{2} \theta} i\left(\frac{n \operatorname{Im} T_{|n-1|}\left(p, q ; e^{i \theta}\right)}{2\left(p^{n-1}-q^{n-1}\right)}-\frac{\operatorname{Im} T_{n+1}\left(p, q ; e^{i \theta}\right)}{p^{n+1}-q^{n+1}}\right) \\
= & \frac{T_{n}(p, q ; 1)}{\sin ^{2} \theta}\left(\frac{n \operatorname{Re} T_{|n-1|}\left(p, q ; e^{i \theta}\right)}{2 T_{|n-1|}(p, q ; 1)}-\frac{\operatorname{Re} T_{n+1}\left(p, q ; e^{i \theta}\right)}{T_{n+1}(p, q ; 1)}\right) \\
& -\frac{U_{|n-1|}(p, q ; 1)}{\sin ^{2} \theta}\left(\frac{n \operatorname{Im} T_{|n-1|}\left(p, q ; e^{i \theta}\right)}{2 U_{|n-2|}(p, q ; 1)}-\frac{\operatorname{Im} T_{n+1}\left(p, q ; e^{i \theta}\right)}{U_{n}(p, q ; 1)}\right) .
\end{aligned}
$$

Various similar formulae are readily obtained for $U_{n}\left(p, q ; e^{i \theta}\right)$. For instance, we get:

Proposition 5 Let $U_{n}\left(p, q ; e^{i \theta}\right)$ be defined by (2.3), then we have

(1)

$$
\begin{aligned}
\cos \theta U_{n-1}\left(p, q ; e^{i \theta}\right)= & \frac{1}{2} U_{n-1}(p, q ; 1)\left(\frac{\operatorname{Re} U_{n}\left(p, q ; e^{i \theta}\right)}{U_{n}(p, q ; 1)}+\frac{\operatorname{Re} U_{n-2}\left(p, q ; e^{i \theta}\right)}{U_{n-2}(p, q ; 1)}\right) \\
& +\frac{1}{2} i T_{n}(p, q ; 1)\left(\frac{\operatorname{Im} U_{n}\left(p, q ; e^{i \theta}\right)}{T_{n+1}(p, q ; 1)}+\frac{\operatorname{Im} U_{n-2}\left(p, q ; e^{i \theta}\right)}{T_{n-1}(p, q ; 1)}\right),
\end{aligned}
$$

(2)

$$
\begin{aligned}
& \left(1-\cos ^{2} \theta\right) U_{n-1}\left(p, q ; e^{i \theta}\right)=\frac{1}{2} U_{n-1}\left(p, q ; e^{i \theta}\right) \\
& -\frac{1}{4} U_{n-1}(p, q ; 1)\left(\frac{\operatorname{Re} U_{n+1}\left(p, q ; e^{i \theta}\right)}{U_{n+1}(p, q ; 1)}+\frac{\operatorname{Re}_{n-3}\left(p, q ; e^{i \theta}\right)}{U_{n-3}(p, q ; 1)}\right) \\
& \quad-\frac{1}{4} i T_{n}(p, q ; 1)\left(\frac{\operatorname{Im} U_{n+1}\left(p, q ; e^{i \theta}\right)}{T_{n+2}(p, q ; 1)}+\frac{\operatorname{Im} U_{n-3}\left(p, q ; e^{i \theta}\right)}{T_{n-2}(p, q ; 1)}\right),
\end{aligned}
$$

(3)

$$
\begin{aligned}
& \cos m \theta T_{n}\left(p, q ; e^{i \theta}\right)=\frac{1}{2} T_{n}(p, q ; 1)\left(\frac{\operatorname{Re}_{n+m}\left(p, q ; e^{i \theta}\right)}{T_{n+m}(p, q ; 1)}+\frac{\operatorname{Re} T_{|n-m|}\left(p, q ; e^{i \theta}\right)}{T_{|n-m|}(p, q ; 1)}\right) \\
& +\frac{1}{2} i U_{n}(p, q ; 1)\left(\frac{\operatorname{Im} T_{n+m}\left(p, q ; e^{i \theta}\right)}{U_{n+m-1}(p, q ; 1)}+\frac{\operatorname{Im} T_{|n-m|}\left(p, q ; e^{i \theta}\right)}{U_{n-m-1}(p, q ; 1)}\right)
\end{aligned}
$$


(4)

$$
\begin{aligned}
& \cos m \theta U_{n-1}\left(p, q ; e^{i \theta}\right) \\
& =\frac{1}{2} U_{n-1}(p, q ; 1)\left(\frac{\operatorname{Re} U_{n+m-1}\left(p, q ; e^{i \theta}\right)}{U_{n+m-1}(p, q ; 1)}+\frac{\operatorname{Re} U_{n-m-1}\left(p, q ; e^{i \theta}\right)}{U_{n-m-1}(p, q ; 1)}\right) \\
& \quad+\frac{1}{2} i T_{n}(p, q ; 1)\left(\frac{\operatorname{Im} U_{n+m-1}\left(p, q ; e^{i \theta}\right)}{T_{n+m}(p, q ; 1)}+\frac{\operatorname{Im} U_{n-m-1}\left(p, q ; e^{i \theta}\right)}{T_{n-m}(p, q ; 1)}\right),
\end{aligned}
$$

(5)

$$
\frac{d U_{n-1}\left(p, q ; e^{i \theta}\right)}{d \theta}=i \frac{(n-1) U_{n}\left(p, q ; e^{i \theta}\right)-p q(n+1) U_{n+1}\left(p, q ; e^{i \theta}\right)}{p e^{i \theta}-q e^{-i \theta}} .
$$

\section{Application}

The generalized Chebyshev polynomials of first kind occurs first in [17], where it was proposed to study the polynomials with one parameter, namely

$$
T_{n}\left(q ; e^{i \theta}\right)=\frac{1}{2}\left(e^{i n \theta}+q^{n} e^{-i n \theta}\right) \quad(q \in[-1,1]) .
$$

Another example is work of Freund and Fisher [10]. In this paper author is concerned with a classical inequality due to Bernstein which estimates the norm of polynomials on any given ellipse in terms of their norm on any smaller ellipse with the same foci. These Bernstein type inequalities are closely connected with certain constrained Chebyshev approximation problems on ellipses. The authors introduce an analogy to the Chebyshev polynomials

$$
T_{k+\frac{1}{2}}(\varphi)=a_{k} \cos \left(k+\frac{1}{2}\right) \varphi+i b_{k} \cos \left(k+\frac{1}{2}\right) \varphi \quad(\varphi \in[-\pi, \pi]),
$$

where

$$
a_{k}=\frac{1}{2}\left(r^{k+\frac{1}{2}}+\frac{1}{r^{k+\frac{1}{2}}}\right), \quad b_{k}=\frac{1}{2}\left(r^{k+\frac{1}{2}}-\frac{1}{r^{k+\frac{1}{2}}}\right) \quad(r \geq 1) .
$$

Finally Freund and Fischer [10] deals with constrained Chebyshev approximation problem of the type

$$
\min _{p \in \prod_{n}: p(c)=1} \max _{z \in E}|P(z)|
$$

Here $\prod_{n}$ denotes the set of all complex polynomials of degree at most $n, E$ is any ellipse in the complex plane, and $c \in \mathbb{C} \backslash E$. In this paper it is showed that the extremal points of $P(z)$ on $E_{r}$ has a form of (2.5). 
Chebyshev polynomials appear in many areas of mathematics. In recent years this interest has often arisen from outside the subject of orthogonal polynomials, after their connection with the class of analytic functions.

Let $\mathcal{A}$ denote the class of holomorphic functions in the unit disk $\mathbb{D}$. The class of typically real functions in $\mathcal{A}$ is denoted by $T_{\mathbb{R}}$. This class is characterized by the condition $\operatorname{Imz} \operatorname{Im} f(z) \geq 0, z \in \mathbb{D}$, and has the integral representation:

$$
T_{\mathbb{R}}=\left\{f: f(z)=\int_{0}^{\pi} \frac{z}{\left(1-z e^{i \theta}\right)\left(1-z e^{-i \theta}\right)} d \mu(\theta), \quad \mu \in \mathcal{P}_{[0, \pi]}\right\},
$$

where $\mathcal{P}_{[0, \pi]}$ denote the set of probability measures on $[0, \pi]$, and was studied by many authors, e.g. $[13,18,19,27,28]$. We suggest [23] the study of the following class of functions.

Definition 5 By $\mathcal{T}^{p, q}$ we denote the class of generalized typically real functions, defined as a class of functions of $f \in \mathcal{A}$, and having an integral representation

$$
f(z)=\frac{1}{2 \pi} \int_{0}^{2 \pi} \frac{z}{\left(1-e^{i \theta} p z\right)\left(1-e^{-i \theta} q z\right)} d \mu(\theta) \quad(z \in \mathbb{D},-1 \leq p, q \leq 1),
$$

where $\mu(\theta)$ is the unique probability measure on the segment $[0,2 \pi]$.

In many classes of analytic functions (e.g. class of typically real functions) Koebe function appears as the extremal. One of the generalization of Koebe function was proposed by Gasper [12]. Namely, he proposed some extension of the L̈̈wner theory and de Brange's inequalities, in which the natural extension of Koebe function is

$$
k_{q}(z)=\frac{z}{(1-z)(1-q z)} \quad(z \in \mathbb{D}),
$$

where $-1 \leq q \leq 1$. We propose an (p, $\mathrm{q})$-extension which is more symmetric, namely

$$
k_{p, q}(z)=\frac{z}{(1-p z)(1-q z)}
$$

where $z \in \mathbb{D},-1 \leq p, q \leq 1$. Due to this context a generalized Chebyshev polynomials is defined for the same range of parameters $-1 \leq p, q \leq 1$. In [16] we investigate mathematical properties of $k_{p, q}$ function, which is associated to the generalized Chebyshev polynomials of the second kind (2.4), as well as to the class of typically real functions that is defined by extended Koebe function (5.1). We approach the problem from both perspectives. By looking for a generating function of orthogonal polynomials and by using orthogonal polynomials as a tool to describe the properties of a class of functions. The motivation for this research is that $k_{p, q}$ function have been found to have many connections with class of $\mathcal{T}^{p, q}$. By considering $k_{p, q}$ function, we gain further insight the connection between class of univalent functions and coefficients problems as well as with orthogonal polynomials. 
One of the most interesting questions in a geometric functions theory is to address the region of variability of the $n$th Taylor coefficient for functions $f$ that belongs to some class of analytic functions. Leading example is the Bieberbach conjecture settled by de Branges in 1985 for the class of normalized and univalent functions $\mathcal{S}$, although corresponding results for important subclasses of $\mathcal{S}$ were established positive much earlier.

In [15] we prove coefficients bounds of functions from the class $\mathcal{T}^{p, q}$. In the case when $p=q=1$ these results become the well known estimates in the class of typically real functions $\mathcal{T}_{\mathbb{R}}$. Using coefficients relations and estimates that hold in that class, we also obtain some sharp coefficients bounds for generalized typically real functions. Orthogonal polynomials $U_{n-1}\left(p, q ; e^{i \theta}\right)$ defined by (2.3) have been used to solve problems related to coefficients bounds in $\mathcal{T}^{p, q}$ class. Namely, from the integral representation (5.1) it follows that the coefficients $f \in \mathcal{T}^{p, q}$ can be represented as

$$
a_{n}=\frac{1}{2 \pi} \int_{0}^{2 \pi} U_{n-1}\left(p, q ; a^{i \theta}\right) d \mu(\theta) .
$$

Therefore, studying the properties of the class $\mathcal{T}^{p, q}$ is a natural problem associated to the general study of orthogonal polynomials defined and considered in this article. These polynomials are interesting themeselves and are the object of interest from the possibilities of their use.

Open Access This article is distributed under the terms of the Creative Commons Attribution 4.0 International License (http://creativecommons.org/licenses/by/4.0/), which permits unrestricted use, distribution, and reproduction in any medium, provided you give appropriate credit to the original author(s) and the source, provide a link to the Creative Commons license, and indicate if changes were made.

\section{References}

1. Akhiezer, N.I.: Orthogonal polynomials on several intervals. Sov. Math. 1, 989-992 (1960)

2. Akhiezer, N.I.: The Classical Moment Problem and Some Related Questions in Analysis. Oliver and Boyed, Edinburgh (1965)

3. Akhiezer, N.I., Tomčuk, J.J.: On the theory of orthogonal polynomials over several intervals. Dokl. Akad. Nauk USSR 138, 743-746 (1961)

4. Andrews, G., Askey, R., Roy, R.: Special Functions. Cambridge University Press, Cambridge (1999)

5. Chebyshev, P.L.: Complete Collected Works. Moscov-Leningrad (1947)

6. Chen, Y., Lawrence, N.: A generalization of the Chebyshev polynomials. J. Phys. A 35, 4651-4699 (2002)

7. Chihara, T.S.: An Introduction to Orthogonal Polynomials. Gordon and Breach, New York (1978)

8. Dickson, L.E.: History of the Theory of Numbers, vol. I, p. 192. Chelsea Publishing Company, New York (1920)

9. Fox, L., Parker, I.B.: Chebyshev Polynomials in Numerical Analysis. Oxford University Press, Oxford (1968)

10. Freund, R., Fisher, B.: On the constrained Chebyshev approximation problem on ellipses. J. Approx. Theory 62, 297-315 (1990)

11. Garcia, A., Stichtenoth, H.: On Chebyshev polynomials and maximal curves. Acta Math. 90, 301-311 (1999) 
12. Gasper, G.: q-Extension of Clausen's formula and of the inequalities used by de Branges in his proof of the Bieberbach, Robertson and Milin conjecture. SIAM J. Math. Anal. 20, 1019-1034 (1989)

13. Golusin, G.M.: On typically-real functions. Math. Sb. 27, 201-217 (1950). (in Russian)

14. Ismail, M.E.: Classical and Quantum Orthogonal Polynomials in One Variable. Cambridge University Press, Cambridge (2005)

15. Kanas, S., Tatarczak, A.: Constrained coefficients problem for generalized typically real functions. (Submitted)

16. Kanas, S., Tatarczak, A.: The generalized typically real functions. (Submitted)

17. Kiepiela, K., Klimek, D.: An extension of Chebyshev polynomials. J. Comput. Appl. Math. 17, 305$312(2005)$

18. Koczan, L., Szapiel, W.: Sur certaines classes des fonctions holomorphes définies par une intègrale de Stieltjes. Ann. UMCS 28, 39-51 (1974)

19. Koczan, L., Zaprawa, P.: Domains of univalence for typically-real odd functions. Complex Var. 48(2), $1-17$ (2003)

20. Krein, M.G.: The ideas of P. L. Chebysheff and A. A. Markov in the theory of limiting values of integrals and their further development. Am. Math. Soc. Transl. Ser. 2, 1-122 (1959)

21. Mason, J.C., Handscomb, D.C.: Chebyshev Polynomials. Chapman and Hall/CRC, London (2003)

22. Morris, A.G., Horner, T.S.: Chebyshev polynomials in the numerical solution of differential equations. Math. Comp. 31, 881-891 (1977)

23. Naraniecka, I., Szynal, J., Tatarczak, A.: An extension of typically-real functions and associated orthogonal polynomials. Ann. UMCS Math. 65, 99-112 (2011)

24. Peherstorfer, F.: On Bernstein-Szegö orthogonal polynomials on several intervals II: orthogonal polynomials with periodic recurrence coefficients. J. Approx. Theory 123-161, 64 (1991)

25. Ray, W.D., Pitman, A.E.N.T.: Chebyshev polynomial and other new approximations to Mills' ratio. Ann. Math. Stat. 34(3), 892-902 (1963)

26. Rivlin, T.J.: Chebyshev Polynomials: From Approximation Theory to Algebra and Number Theory. Wiley, New York (1990)

27. Robertson, M.S.: On the coefficient of typically-real functions. Bull. Am. Math. Soc. 41, 565-572 (1935)

28. Rogosinski, W.: Über positive harmonische Entwicklungen und typisch-reelle Potenzreihen. Math. Zeit. 35, 93-121 (1932)

29. Shapiro, W.: A combinatorial proof of a Chebyshev polynomial identity. Discret. Math. 34, 203-206 (1981)

30. Shparlinskii, I.E.: Generalization of Chebyshev polynomials. Sib. Math. J. 31(1), 183-185 (1990)

31. Szegö, G.: Orthogonal Polynomials. American Mathematical Society, Providence (1975)

32. Tomčuk, J.J.: Orthogonal polynomials over a system of intervals on the number line. Zap. Mekh. Mat. Fak. Kharkov Mat. Oshch. 29, 93-128 (1964)

33. Witula, R., Slota, D.: On modified Chebyshev polynomials. J. Math. Anal. Appl. 324(1), 321-343 (2006) 